\title{
TI.91.1
}

\section{CSI2 Working Group}

- PDF: security-topics-update-CSI2-V4.pdfsecurity-topics-update-CSI2-V4.pdf

- Text: security-topics-update-CSI2-V4.txtsecurity-topics-update-CSI2-V4.txt

\section{More Information}

\begin{tabular}{|l|l|}
\hline Repository ID & TI.91.1 \\
\hline Persistent URL & http://doi.org/10.26869/TI.91.1 \\
\hline Title & CSI2 Working Group \\
\hline Authors & \\
\hline Sponsor & SALSA \\
\hline Review & \\
\hline Status & Legacy \\
\hline Publish Date & April, 2006 \\
\hline DOI & $10.26869 /$ TI.91.1 \\
\hline Signature & \\
\hline Deprecated & No \\
\hline Future Review & \\
\hline Supersedes & \\
\hline Format & PDF, Text \\
\hline Related Docs & \\
\hline Development Location & \\
\hline IP Framework & \\
\hline Subject Tags & middlewarerescue \\
\hline Notes & \\
\hline
\end{tabular}

